\title{
The hypothalamic-pituitary-adrenal axis in anxiety and panic
}

\author{
Frederico Guilherme Graeff and Hélio Zangrossi Junior \\ Universidade de São Paulo, Brazil
}

\begin{abstract}
This review article focuses on the differential activation of the hypothalamic-pituitary-adrenal (HPA) axis in generalized anxiety and panic. The results of experimental studies that assayed adrenocorticotropic hormone, cortisol and prolactin show that real-life panic attacks as well as those induced by selective panicogenic agents, such as lactate and carbon dioxide, do not activate the HPA axis. Accordingly, experiments carried out in two animal models of panic, namely electrical stimulation of the dorsal periaqueductal gray matter of the rat and the escape from the open arm of the elevated T maze, have shown that in neither case stress hormones are increased in the plasma. Also in humans, reported results have shown that neither cortisol nor prolactin levels were increased following simulated public speaking, an experimental task that has been related to panic, in either healthy volunteers or patients with panic disorder diagnosis. Therefore, although the panic attack causes a major sympathetic stimulation, it has little effect on the HPA axis. In contrast, anticipatory or generalized anxiety activates both the HPA and the sympatho-adrenal axes. Keywords: stress hormones, generalized anxiety, panic, simulated public speaking, periaqueductal gray matter, elevated T maze.
\end{abstract}

Received 12 February 2010; received in revised form 12 April 2010; accepted 12 April 2010. Available on line 26 June 2010

\section{Introduction}

The concept of stress is based on the observation that different kinds of physical or psychological demands on the organism elicit the same set of bodily changes, the so called General Adaptation Syndrome (GAS) (Selye, 1936; Ursin \& Olff, 1993). However, Hans Selye (1974) himself acknowledged that the original name was not the best one: "...when I introduced the word stress ..., my English was not yet good enough for me to distinguish between the words stress and strain. It was not until several years later that the British Medical Journal called my attention ... that according to Selye stress is its own cause. Actually I should have called my phenomenon the strain reaction and that which causes it 'stress', which would parallel the use of these terms in physics. However, ... biological stress in my sense of the word was so generally accepted in various languages that I could not have redefined it. Hence, I was forced to create a neologism and

Frederico Guilherme Graeff, Departamento de Neurociências e Ciências Comportamentais, Faculdade de Medicina de Ribeirão Preto, Universidade de São Paulo, Brazil. Hélio Zangrossi Junior, Departamento de Farmacologia, Faculdade de Medicina de Ribeirão Preto, Universidade de São Paulo, Brazil. Correspondence regarding this article should be directed to: Frederico G. Graeff, Departamento de Neurociências e Ciências Comportamentais, Faculdade de Medicina de Ribeirão Preto, USP, Hospital das Clínicas, 3o andar, Avenida dos Bandeirantes 3900, Ribeirão Preto, SP, Brazil, 14048-900. Phone: + 55-16-3602-2607. Fax: +55-163602-2544. E-mail: hegog@hotmail.com introduce the word stressor, for the causative agent, ... retaining stress for the resulting condition". (Selye, 1974, as cited in Pacák \& Palkovits, 2001).

The most characteristic stress response is the release of the adrenocorticotropic hormone (ACTH) and corticoids (cortisol in humans and cortisone in the rat) into the blood stream as a result of activation of the hypothalamic-pituitary-adrenal (HPA) axis. In addition to the HPA axis, acute stress also activates the sympathetic division of the neurovegetative nervous system as part of the fight/flight reaction, or emergency response, defined by Walter Cannon (1914). As a result, noradrenaline is released from peripheral sympathetic nerve fibers in different tissues, and adrenaline (also some noradrenaline), from the adrenal medulla into the blood stream.

More recently, McEwen has introduced the concept of allostasis, that is, maintaining stability through behavioral change, as a fundamental process through which animals actively adjust to predictable or unpredictable stressors; allostatic load refers to the cumulative cost of allostasis to the organism, with allostatic overload being a state in which pathology can occur (McEwen \& Wingfield, 2003).

Stressors may be physical, such as tissue damage or extreme changes in temperature, but may also be psychological. As to the latter, reported results have consistently shown that the HPA axis and sympathetic nervous system are activated by novelty or cues that signal the delivery of punishment or the withholding of an expected reward (frustration), thus generating anticipatory anxiety. The neural circuits that mediate 
the neuroendocrine responses to psychological stressors include the cortical activation of the basolateral nucleus of the amygdala, which in turn activates its central nucleus. The message is then conveyed to hypothalamic neurons by different pathways: a direct one, an indirect one, through the bed nucleus of the stria terminalis, and still another one, through brainstem serotonin (5-HT) and catecholamine-containing neurons. Neurons of the hypothalamic paraventricular nucleus secrete the corticotropic releasing hormone $(\mathrm{CRH})$ into the portal circulation of the pituitary gland. In the anterior pituitary, CRH stimulates ACTH-secreting cells that release ACTH into the blood stream. ACTH acts on the adrenal cortex promoting cortisol release into the blood stream. In addition to ACTH, prolactin is consistently released at the anterior pituitary in stressful conditions (Van der Kar \& Blair, 1999).

To test the GAS hypothesis, Miklós Palkovits and coworkers have used five different stressors: immobilization, hemorrhage, cold exposure, pain, or hypoglycemia. With the exception of immobilization stress, these stressors also differed in their intensities. Their results showed marked heterogeneity of neuroendocrine responses to various stressors and that each stressor has a neurochemical "signature." By examining changes of Fos immunoreactivity in various brain regions upon exposure to different stressors, they also described stressor-specific pathways and circuits. As a result, they have defined stress as a state of threatened homeostasis (physical or perceived threat to homeostasis) that triggers stimulus-specific adaptive compensatory responses. The adaptive response reflects the activation of specific central circuits and is genetically and constitutionally programmed and constantly modulated by environmental factors (Pacák $\&$ Palkovits, 2001). This view may help to understand the seeming paradox on panic attacks and the HPA axis that is discussed in the following section.

\section{Defense-related emotions}

Anxiety, fear and panic are emotions related to threat. The distinction between anxiety and fear has not always been clear, but the ethoexperimental approach developed by Robert and Caroline Blanchard (Blanchard $\&$ Blanchard, 1988) has provided a sound criterion based on the systematic study of animal defensive strategies against predators. From this perspective, anxiety is the emotion related to risk-assessment behavior that is evoked in situations when the danger is uncertain (potential threat); either because the context is novel or because the danger stimulus (e.g., a predator) had been present in the past, but is no longer in the environment. The notion of risk assessment overlaps with that of conflict, which is a cornerstone of anxiety research. In contrast, fear is related to defensive strategies that occur in response to actual danger that is at a certain distance from the prey (distal threat). In this case, the animal either evades the situation, whenever an escape route is available or becomes tensely immobilized (freezing), when there is no way out. Finally, panic corresponds to the vigorous flight reaction evoked by very close danger (proximal threat), such as an approaching predator or by acute cutaneous pain. Complete immobility also occurs in response to proximal danger, as well as defensive fight, which occurs when flight is impossible. Nevertheless, the latter strategy relates to rage, rather than to panic (see also Gray \& McNaughton, 2000, McNaughton \& Corr, 2004). Concerning psychopathology, it has been suggested that the same neurobiological processes that regulate anticipatory anxiety are involved in generalized anxiety disorder (GAD); the ones that control fear, in phobic disorders, and those organizing proximal defense, in panic disorder (PD) (Deakin \& Graeff, 1991).

According to the DSM IV classification of psychiatric disorders (American Psychiatric Association, 1994), PD is characterized by the occurrence of panic attacks, in which feelings of extreme fear and dread strike unexpectedly and repeatedly, accompanied by intense physiological symptoms. Over time, anticipatory anxiety about having a further attack and avoidance of places where having an attack is embarrassing develop. The extreme form of avoidance is agoraphobia, in which case the person is afraid of leaving home alone. In contrast, the main symptom of GAD is exaggerated worry and tension over every day events and decisions, lasting for at least six months. In addition to the different clinical manifestations, the pharmacological profile of these disorders also differs: GAD is rapidly ameliorated by anxiolytic drugs, PD by chronic administration of antidepressants (Nutt, 2005).

Emotions, either normal or abnormal, are manifested in both psychological (cognitive, affective, behavioral) and physiological (neurovegetative, neuroendocrine) domains. The question that is central to the present article is whether anxiety/GAD and panic/PD differ qualitatively or only quantitatively as to the activation of the HPA axis.

\section{Neuroendocrinology of panic and anxiety}

A naturally occurring PA can be considered as a traumatic stressor (McNally \& Lukach, 1992). Assuming the GAS hypothesis-there is single stereotyped hormonal response to all kinds of stressors -, it is expected that the HPA axis would be much more activated by a panic attack than by anxiety. Yet, the majority of the reported results indicate that the HPA axis is little affected by PAs. Thus, a review of the literature on stress hormone responses during PAs (Graeff, Garcia-Leal, Del-Ben, \& Guimarães, 2005) showed that real-life PAs as well as those induced by selective panicogenic agents, such 
as lactate and carbon dioxide, do not activate the HPA axis. Agonists of the colecystokinin receptor B, such as the colecystokinin-4 peptide (CCK-4) and pentagastrin, increase stress hormones regardless of the occurrence of a PA and thus seem to activate the HPA axis directly. The benzodiazepine antagonist flumazenil does not increase stress hormones, but this agent does not reliably induce a PA. Pharmacological agents that increase anxiety in both normal subjects and panic patients, such as yohimbine, 1-(m-chlorophenyl) piperazine (mCPP), fenfluramine and caffeine and mCPP consistently raised stress hormone levels.

The above evidence indicates that natural PAs fail to activate the HPA axis, in contrast to anticipatory anxiety. Yet, the HPA axis responsivity to mild stress seems to be normal in children of parents with PD (Battaglia et al., 1997), but in adult PD patients, there is a reduced response to mild stress (Stones, Groome, Perry, Hucklebridge, \& Evans, 1999) as well as to a combined dexamethasone-corticotropin-releasing hormone challenge (Schreiber, Lauer, Krumrey, Holsboer, \& Krieg, 1996). Therefore, the HPA axis may become progressively desensitized after repeated exposure to PAs (Van Duinen, Schruers, Maes, \& Griez, 2006). Another possibility is the uncoupling of the HPA axis and the noradrenergic-hypothalamic-pituitaryadrenal axis, which is always markedly activated during the PA (Coplan et al., 1995). In contrast to PD, both the HPA axis and the sympatho-adrenal axis are activated by anticipatory anxiety (Mason, 1975). In acute anxiety, the activation of the HPA axis is adaptive, since among other things corticoids seem to reduce perceived fear by impairing memory retrieval of emotionally arousing information (Soravia et al., 2006).

Experimental tests of the hypothesis that brain systems involved in PA do not activate the HPA axis have been conducted in animal models of anxiety and in human experimental anxiety trials.

\section{Tests with animal models of anxiety}

Since the PAG has been implicated in the PA, electrical or chemical stimulation of this brain structure has been used as an animal model of panic (see Graeff \& Zangrossi Jr., 2002). In one of these models, developed by L.C. Schenberg and coworkers, behavioral items evoked by electrical stimulation of the dorsal PAG of the rat, such as freezing, running, galloping, and so on were measured. The most important finding was that PAG-evoked galloping was selectively attenuated by clinically-effective panicolytics, given in a dose-regimen and time-course not far from those of panic therapy. Particularly, galloping was either attenuated or virtually abolished by 21-day administration of the 5-HT reuptake inhibitors clomipramine and fluoxetine. Treatments that are clinically ineffective on PD, such as the acute administration of these antidepressants and diazepam, or the acute and 10-day administration of buspirone, did not attenuate the PAG- evoked behaviors. In addition, PAGevoked galloping was selectively facilitated by peripheral injections of pentylenetetrazole, a putative panicogen in humans (Schenberg, Bittencourt, Sudre, \& Vargas, 2001; Schenberg, Capucho, Vatanabe, \& Vargas, 2002; Vargas \& Schenberg, 2001). These pharmacological results indicate that galloping induced by DPAG electrical stimulation is a reliable panic model.

Using this model, Schenberg, Dos Reis, Ferreira Póvoa, Tufik and Silva (2008) have examined whether the plasma levels of stress hormones are changed following PAG-evoked behaviors. The obtained results have shown that $\mathrm{ACTH}$ and prolactin plasma concentrations remain unaltered following the PAG-evoked defensive reactions. Not even a 5 minute electrical stimulation of the dorsal PAG at the flight threshold intensity significantly changed ACTH plasma level. The activity of the HPA axis has also been assessed in another experimental model that associates flight/escape behavior with PA, the elevated T-maze (for a review of this model see Graeff \& Zangrossi, 2002; Pinheiro, Zangrossi, Del-Ben, \& Graeff, 2007). As this test also allows the measurement of inhibitory avoidance, it has been possible to draw direct comparisons on the activity of the axis after expression of a PA or a GAD-related defensive response. The elevated T-maze is derived from the elevated plus-maze by sealing the entrance to one of its enclosed arms. As a result, the T-maze consists of three arms of equal dimension (50 x $12 \mathrm{~cm}$ ) elevated $50 \mathrm{~cm}$ from the floor. One of these arms is enclosed by lateral walls ( $40 \mathrm{~cm}$ high) and stands perpendicular to the two opposite open arms. When placed at the end of the enclosed arm, the rat does not see the open arms until it pokes its head beyond the walls of the closed arm. Being on the open arm seems to be an aversive experience, since rats have an innate fear of height and openness (Treit, Menard, \& Royan, 1993). This would allow the animal to learn inhibitory avoidance if repeatedly placed inside the enclosed arm to explore the maze. On the other hand, when the rat is placed at the end of one of the open arms it can move towards the closed arm, presumably performing an escape response. The pharmacological exploration of the T-maze showed that these two defensive tasks are differently affected by drug treatments. Thus, compounds representative of three classes of anxiolytics - namely the agonist of BZD receptors diazepam, the serotonin 5- $\mathrm{HT}_{1 \mathrm{~A}}$ agonist buspirone, and the nonselective 5- $\mathrm{HT}_{2}$ antagonist ritanserin - have been shown to selectively impair inhibitory avoidance while leaving escape unchanged. These results are compatible with the view that inhibitory avoidance relates to GAD. In contrast, the escape task is insensitive to different classes of anxiolytics, and is impaired by chronic, but not acute administration of imipramine, clomipramine and fluoxetine, drugs that are used to treat PD. 
Figure 1 (upper panel) shows the latency to leave the arms of the elevated T-maze on 3 consecutives trials. It can be seen that the latency to leave the enclosed arm increases across trials, indicating the acquisition of inhibitory avoidance. Latency to leave one of the T-maze open arms (escape) or one of the arms of a maze consisting of three enclosed arms (control) did not significantly change across trials. As shown in the lower panel of Figure 1, plasma levels of corticosterone are significantly higher in animals that acquired inhibitory avoidance, once again indicating the differential activation of the HPA axis on GAD and PA-associated behaviors.

\section{Tests with human experimental anxiety}

The main psychological procedures for inducing experimental anxiety in human beings for pharmacological studies have been the aversive conditioning of the skin conductance response and the simulated public speaking (SPS) tests. While the drug
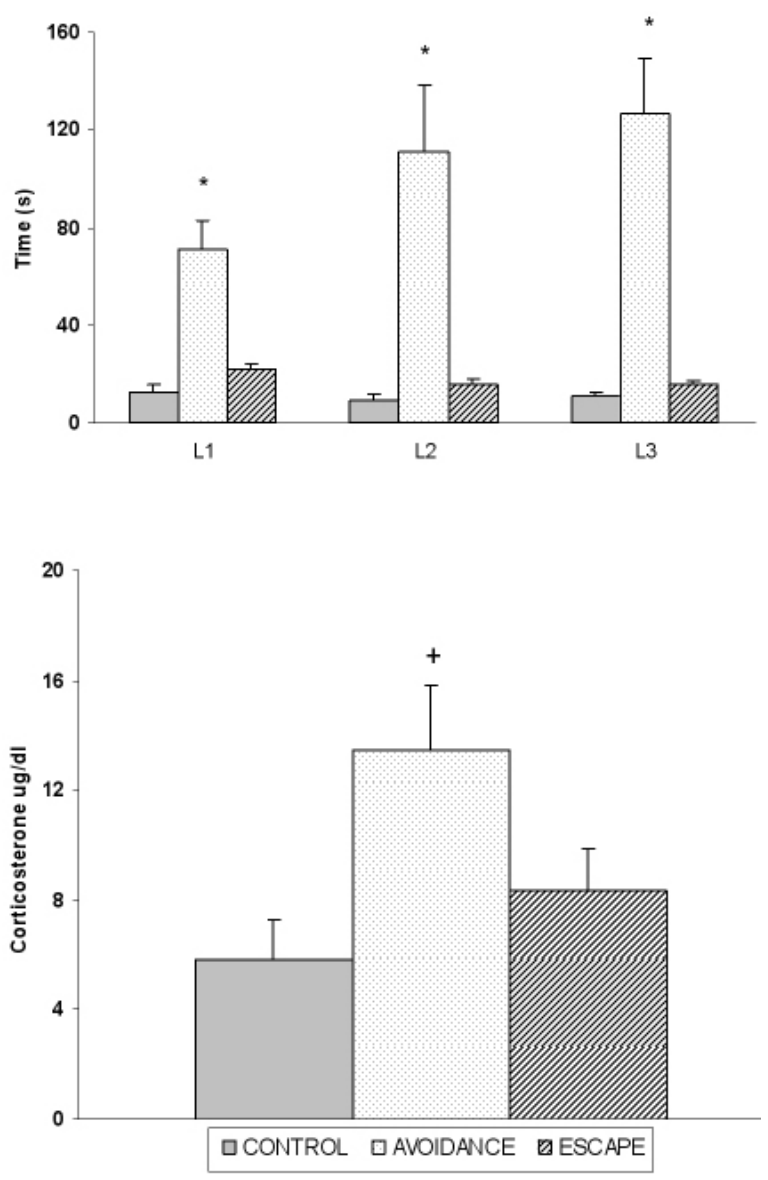

Figure 1. Upper panel: Bar chart comparing latencies (mean \pm SEM) taken by rats to withdraw from an enclosed arm of an enclosed T-maze (control) or from the enclosed (avoidance) or open (escape) arm of the elevated T-maze, in three consecutive trials (L1, L2 and L3). ${ }^{*} P<.05$ (Duncan's test) compared with all other groups. Lower panel: Mean \pm SEM of plasma corticosterone levels measured immediately after the performance of control, avoidance and escape tasks. ${ }^{+} P<.05$ (Duncan's test) compared with the control group. profile of the former is similar to that of GAD, SPS has a pharmacological profile that resembles PD and social anxiety disorder, and is believed to mobilize the same neural network that is involved in these disorders (for a review, see Graeff, Parente, Del-Ben, \& Guimarães, 2003). As a consequence, two studies have been conducted by our research group to investigate whether SPS would affect HPA axis functioning.

In the SPS test, each participant is requested to prepare a speech and talk in front of a videocamera, the performance being recorded on videotape. Anxiety and other subjective states are evaluated by a psychometric instrument, the Visual Analog Mood Scale (VAMS). Also, bodily symptoms related to anxiety are assessed by the Bodily Symptom Scale (BSS). In the first endocrinological study (Garcia-Leal et al., 2005), the participants were divided into three groups: 18 symptomatic panic patients, 16 nonsymptomatic, drugtreated panic patients, and 17 healthy controls. Along the experimental session, the VAMS anxiety index and the total score of the BSS were higher in symptomatic patients than in controls, nonsymptomatic patients lying in between. In every group, the level of salivary cortisol was high at the beginning of the experimental session, and decreased after 70 minutes. This fall parallels the decrease in the VAMS anxiety factor and in BSS ratings, and appears to reflect habituation of the initial, anticipatory anxiety evoked by exposure to the new and potentially threatening laboratory environment. Accordingly, there has been a positive correlation between the initial level of cortisol and VAMS anxiety scores for the three groups, taken together. Preparation and performance of speech raised the VAMS anxiety index and BSS scores to the initial levels, but failed to increase salivary cortisol measured along 60 minutes, starting at the end of the speech (Figure 2). Therefore, SPS does not seem to activate the HPA axis, in contrast to anticipatory anxiety.

The second study was aimed at evaluating the effects of escitalopram, a very potent and selective 5-HT reuptake inhibitor, on SPS (Garcia-Leal, Del-Ben, Leal, Graeff, \& Guimarães, 2009). Healthy male volunteers received, in a double-blind randomized design, placebo $(\mathrm{n}=12), 10(\mathrm{n}=17)$ or $20(\mathrm{n}=14) \mathrm{mg}$ of escitalopram, two hours before the test. Both doses of escitalopram did not affect the increase in VAMS anxiety scores determined by speech preparation or performance, but prolonged the rise induced by SPS. The most important results for the present argument are that the test itself did not significantly change cortisol plasma levels; neither did it change the levels of prolactin. Therefore, once more SPS failed to activate the HPA axis. However, under the highest dose of escitalopram, cortisol and prolactin increased immediately after the SPS (Figure 3 ). The last result suggests that 5-HT modulates the release of stress hormones. 


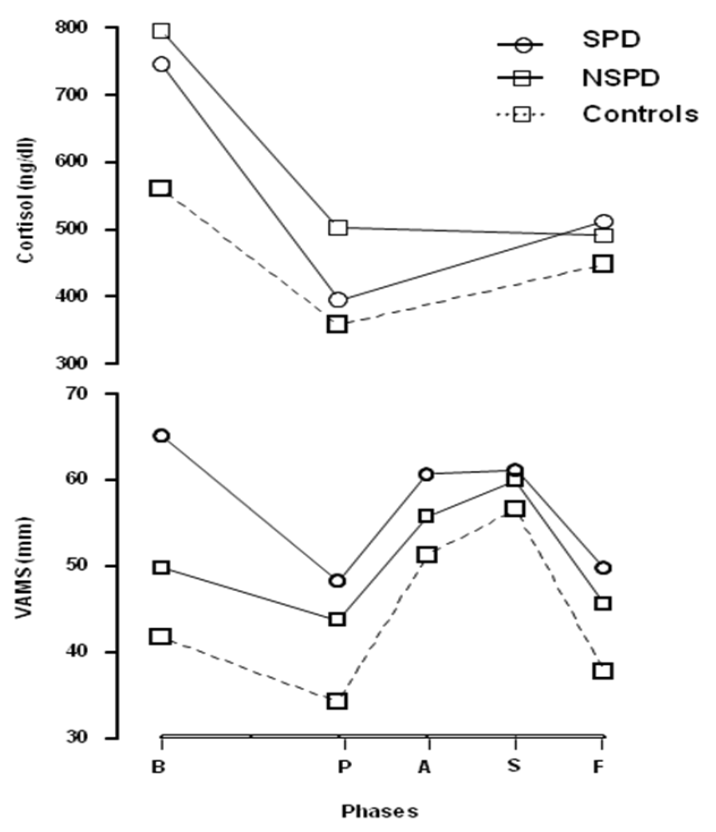

Figure 2. Upper panel: Mean salivary cortisol concentration before and after the simulated public speaking (SPS) test, measured in 18 symptomatic panic patients, 16 nonsymptomatic patients and 17 healthy controls. The initial measure was taken 25 minutes after the subject arrived in the laboratory. The last measure is the average of the highest value for each participant along 60 minutes starting at end of the speech. Measurements were taken immediately, 15, 30 and 60 minutes after the speech. Lower panel: Mean VAMS anxiety factor along the experimental session in the same subjects. Session phases are: B - initial, P - pre-test, A - speech preparation, $\mathrm{S}$ - speech performance, $\mathrm{F}$ - final.

In contrast to the above SPS test, a similar procedure known as the Trier Social Stress Test (TSST), in which the participant faces an audience and is requested to perform arithmetical calculations (Kirchbaum, Pirke, \& Hellhammer, 1993), has been shown to increase salivary cortisol in normal volunteers, but fail to do so in PD patients (Petrowski, Herold, Joraschky, Wittchen, \& Kirschbaum, 2010). These results further support the view that PD patients lack cortisol responsivity to acute uncontrollable psychosocial stress. This unresponsiveness of the HPA axis seems to be rather specific, since in the mentioned study a normal cortisol awakening response in the morning has been recorded in the same patients.

\section{Conclusions}

The preceding evidence indicates that anxiety and panic are qualitatively different emotional states, which are related to the defense reactions to potential and proximal threat, respectively. Equally different are the related pathologies, GAD and PD, which differ both in their symptomatology and in the response to pharmacotherapy. Thus, specific neurobiological processes underlie each of these conditions. In regard to stress hormones, the analyzed data suggest that while anxiety activates both the HPA and the sympathoadrenal axes, the panic attack causes major sympathetic activation, but has little effect on the HPA axis.

At a more general level, the distinction between the hormonal responses to anxiety as compared to panic supports the view held by Palkovits that there are specific adaptive responses to each types of stress. This casts doubts on the existence of a GAS and, therefore on Selyes' original concept of stress itself. Even the alternative concept of stress as "a state of threatened to homeostasis" (Pacák \& Palkovits, 2001) may be redundant to Cannon's notion of homeostasis, which implies that the organism tends to keep its internal state (Claude Bernard's milieu intérieur) within narrow limits through specific adaptive responses that tend to correct any provoked imbalance. Therefore, in spite of its major impact on research (heuristic value), on common knowledge and on every day life, the explanatory value of the concept of stress needs reevaluation.
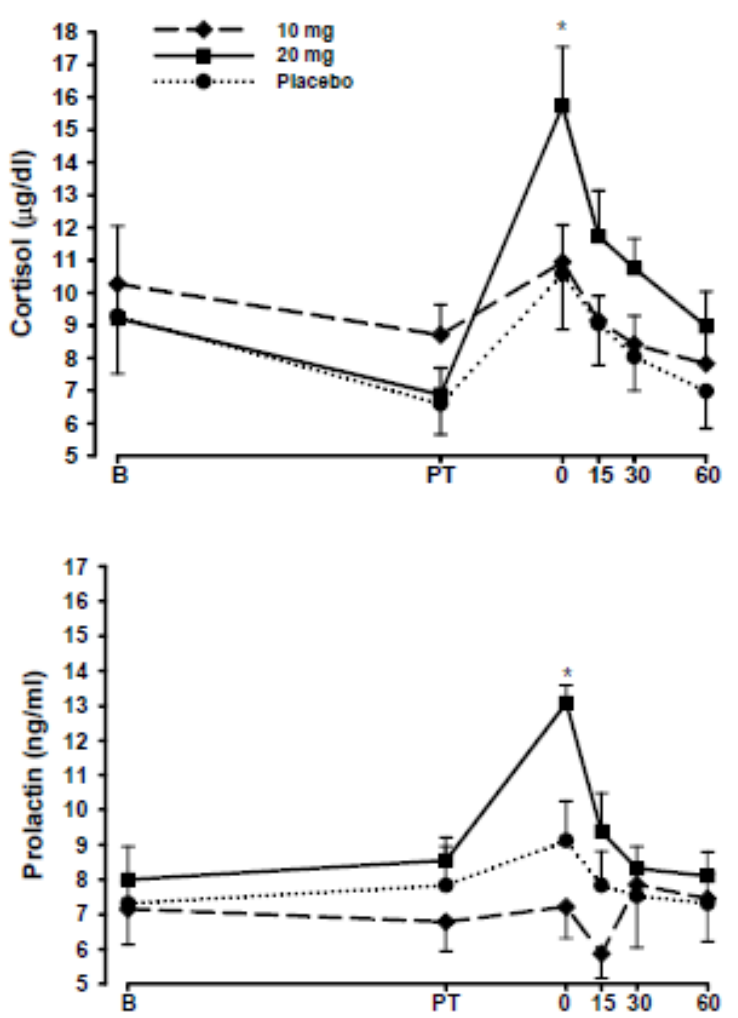

Figure 3. Plasma cortisol and prolactin levels along the SPS experimental session, measured in 37 healthy male volunteers treated with an acute oral doses of $10 \mathrm{mg}(\mathrm{n}=14)$ or 20 $\mathrm{mg}(\mathrm{n}=11)$ of escitalopram, compared to placebo $(\mathrm{n}=12)$. Measurements were taken 60 minutes after the arrival in the laboratory (B), two hours after drug or placebo intake and before the speech $(\mathrm{P})$, as well as immediately (0), and 15 , 30 and 60 minutes after the end of the speech. *Significantly different from PT and from other two groups. 


\section{Acknowledgments}

F. G. Graeff is recipient of research fellowships from CNPq and FAEPA - Hospital das Clínicas FMRPUSP. The authors thank Tatiana Longo Borges for allowing the reproduction of Figure 1 in the present review.

\section{References}

American Psychiatric Association (1994). Diagnostic and Statistical Manual of Mental Disorders. Washington, DC: APA Press.

Battaglia, M., Bajo, S., Strambi, L.F., Brambilla, F., Castronovo, C., Vanni, G.. \& Bellodi, L. (1997). Physiological and behavioral responses to minor stressors in offspring of patients with panic disorder. Journal of Psychiatry Research, 31(3), 365-376.

Blanchard, D.C., \& Blanchard, R.J. (1988). Ethoexperimental approaches to the biology of emotion. Annual Reviews of Psychology, 39(1), 43-68.

Cannon, W.B. (1914). The emergency function of the adrenal medulla in pain and the major emotions. American Journal of Physiology, 33(2), 356-372.

Coplan, J.D., Pine, D., Papp, L., Martinez, J., Cooper, T., Rosenblum, L.A., \& Gorman, J.M. (1995). Uncoupling of the noradrenergichypothalamic-pituitary-adrenal axis in panic disorder patients. Neuropsychopharmacology, 13(1), 65-73.

Deakin, J.F.W., \& Graeff, F.G. (1991). 5-HT and mechanisms of defense. Journal of Psychopharmacology, 5(4), 305-315.

Garcia-Leal, C., Del-Ben, C.M., Leal, F.M., Graeff, F.G., \& Guimarães, F.S. (2009). Escitalopram prolonged fear induced by simulated public speaking and released hypothalamic-pituitary-adrenal axis activation. Journal of Psychopharmacology, Feb 27. [Epub ahead of print]

Garcia-Leal, G., Parente, A.C.B.V., Del-Ben, C.M., Guimarães, F.S., Moreira, A.C., Elias, L.L.K., \& Graeff, F.G. (2005). Anxiety and salivary cortisol in symptomatic and nonsymptomatic panic patients and healthy volunteers performing simulated public speaking. Psychiatry Research, 133(2-3), 239-252.

Graeff, F.G., \& Zangrossi Jr., H. (2002). Animal models of anxiety disorders. In H. D'haenen, J.A. Den Boer, H. Westenberg, \& P. Willner (Eds.), Textbook of Biological Psychiatry (pp. 879-893). London: John Wiley \& Sons.

Graeff, F.G., Parente, A., Del-Ben, C.M., \& Guimarães, F.S. (2003). Pharmacology of human experimental anxiety. Brazilian Journal of Medical and Biological Research, 36(4), 421-432.

Graeff, F.G., Garcia-Leal, C., Del-Ben, C.M., \& Guimarães, F.S. (2005). Does the panic attack activate the hypothalamic-pituitary-adrenal axis? Anais da Academia Brasileira de Ciências, 77(3), 1-15.

Gray, J.A., \& McNaughton, N. (2000). The neuropsychology of anxiety. Oxford: Oxford University Press.

Kirschbaum, C., Pirke, K.M., \& Hellhammer, D.H. (1993). The ‘Trier Social Stress Test' - a tool for investigating psychobiological stress responses in a laboratory setting. Neuropsychobiology, 28(1-2), 76-81.

McNaughton, N., \& Corr, P.J. (2004). A two-dimensional neuropsychology of defense: fear/anxiety and defensive distance. Neuroscience and Biobehavioral Reviews, 28(3), 285-305.

Mason, J.W. (1975). A historical review of stress. Journal of Human
Stress, 1(1), 6-12.

McEwen, B.S., \& Wingfield, J.C. (2003). The concept of allostasis in biology and biomedicine. Hormones and Behavior, 43(1), 2-15.

McNally, R.J., \& Lukach, B.M. (1992). Are panic attacks traumatic stressors? American Journal of Psychiatry, 149(6), 824-826.

Nutt, D.J. (2005). Overview of diagnosis and drug treatments of anxiety disorders. CNS Spectrums, 10(1), 49-56.

Pacák K., \& Palkovits M. (2001). Stressor specificity of central neuroendocrine responses: implications for stress-related disorders. CNS Spectrums, 22(4), 502-548.

Petrowski, K., Herold, U., Joraschky, P., Wittchen, H.-U., \& Kirschbaum, C. (2010). A striking pattern of cortisol nonresponsiveness to psychosocial stress in patients with panic disorder with concurrent normal cortisol awakening responses. Psychoneuroendocrinology, 35(3), 414-421.

Pinheiro, S.H., Zangrossi Jr., H, Del-Ben, C.M., \& Graeff, F.G. (2007). Elevated mazes as animal models of anxiety: effects of serotonergic agents. Anais da Academia Brasileira de Ciências, 79(1), 71-85.

Schenberg, L.C., Bittencourt, A.S., Sudre, E.C., \& Vargas, L.C. (2001). Modeling panic attacks. Neuroscience and Biobehavioral Reviews, 25(7-8), 647-659.

Schenberg, L.C., Capucho, L.B., Vatanabe, R.O., \& Vargas, L.C. (2002). Acute effects of clomipramine and fluoxetine on dorsal periaqueductal grey-evoked unconditioned defensive behaviours of the rat. Psychopharmacology, 159(2), 138-144.

Schenberg, L.C., Dos Reis, A.M., Ferreira Póvoa, R.M., Tufik, S., \& Silva, S.R. (2008). A panic attack-like unusual stress reaction. Hormones and Behavior, 54(5), 584-591.

Schreiber, W., Lauer, C.J., Krumrey, K., Holsboer, F., \& Krieg, J.C. (1996). Dysregulation of the hypothalamicpituitary-adrenocortical system in panic disorder. Neuropsychopharmacology, 15(1), 7-15.

Selye, H. (1936). A syndrome produced by diverse nocuous agents. Nature, 138(1), 32 .

Selye, H. (1974). Stress without distress. New York: New York American Library.

Soravia, L.M., Heinrichs, M., Aerni, A., Maroni, C., Schelling, G., Ehlert, U., Roozendaal, B., \& de Quervain, D.J. (2006). Glucocorticoids reduce phobic fear in humans. Proceedings of National Academy of Science USA, 103(14), 5585-5590.

Stones, A., Groome, D., Perry, D., Hucklebridge, F., \& Evans, P. (1999). The effect of stress on salivary cortisol in panic disorder patients. Journal of Affective Disorders, 52(1), 197-201.

Treit, D., Menard, J., \& Royan, C. (1993). Anxiogenic stimuli in the elevated plus-maze. Pharmacology Biochemistry and Behavior, 44(2), 463-9.

Ursin, H., \& Olff, M. (1993). The stress response. In S. Stanford \& P. Salmon (Eds.), Stress: from Synapse to Syndrome (pp. 3-22). New York: Academic Press.

Van de Kar, L.D., \& Blair, M.L. (1999). Forebrain pathways mediating stress-induced hormone secretion. Frontiers in Neuroendocrinology, 20(1), 1-48.

Van Duinen, M.A., Schruers, K.R., Maes, M., \& Griez, E.J. (2006). CO2 challenge induced HPA axis activation in panic. The International Journal of Neuropsychopharmacology, 10(6), 797-804.

Vargas, L.C., \& Schenberg, L.C. (2001). Long-term effects of clomipramine and fluoxetine on dorsal periaqueductal grey-evoked innate defensive behaviors of the rat. Psychopharmacology, 155(3), 260-268. 